\title{
The Effects of Entrance Examination Stress on Oral Health Behavior and Subjective Oral Health in Female High School Students
}

\author{
Kyung-Yi Chung ${ }^{\dagger}$ \\ Department of Dental Hygiene, Honam University, Gwangju 62399, Korea
}

\begin{abstract}
Background: The purpose of this study is to investigate the effects of entrance exam stress on oral health behaviors and subjective oral health status in female high school students.

Methods: A self-reported questionnaire was administered to 216 female high school students in the Gwangju area. We performed an independent t-test, one-way analysis of variance (ANOVA), Pearson's correlation coefficient, and multiple regression analysis using the SPSS/WIN 21.0 program.

Results: The average of the entrance exam stress was 2.73, and among the sub-areas, the tension for exam/poor stress was the highest with 3.21. As a result of analysis of general characteristics and subjective oral health status, oral mucosal disease was lower in family income level $(p<0.05)$, bad breath was significantly higher with lower academic performance $(p<0.001)$ and family income level $(p<0.05)$. As a result of oral health behavior and subjective oral health status analysis, dental caries has more than 4 times of caries snacks (per week) $(p<0.05)$, bad breath was found to be more perceived when the average number of daily brushing was 2 or less $(p<0.01)$. Factors affecting subjective oral health status were that dental caries was a patients pressure stress $(\beta=0.202)$; temporomandibular disorder $(\beta=0.227)$, xerostomia $(\beta=0.342)$, and oral mucosal disease $(\beta=0.190)$ were insufficient spare time; bad breath was academic performance (lower) $(\beta=0.231)$ and insufficient spare time $(\beta=0.184)$. There was a statistically significant positive correlation between the subjective oral health status and the subarea of entrance exam stress, excluding future uncertainty stress.

Conclusion: It is considered that oral health education should be conducted to prevent oral diseases for students with high parental pressure and insufficient spare time stress as well as finding practical ways to reduce entrance stress.
\end{abstract}

Key Words: Female, High school, Oral health, Stress, Subjective

\section{Introduction}

The period of adolescence is filled with many physiological changes and physical developments, and oral health behaviors and awareness formed during this period play a critical role in the acquisition of oral health care capacity throughout adulthood and senescence ${ }^{1)}$. However, as most adolescents have poor awareness regarding oral health care, they are susceptible to oral diseases such as dental caries and periodontal diseases.

In the survey of high school students, the highest percentage of $64.9 \%$ responded that on average, they brushed their teeth ' $\leq 2$ times', and regarding whether they frequently enjoy sugary foods like snacks and coke, $52.8 \%$ responded 'moderately' while $30.9 \%$ responded 'frequently'. In all, $74 \%$ of students responded that they had not been to a dental clinic in the recent year. In addition, the students who responded "daily brushing $\leq 2$ times," the number of decayed teeth was $1 \sim 2$ in $90 \%$ and $\geq 3$ in $88.4 \%$, while among those who frequently enjoy tooth-decaying snacks, the number of decayed teeth was $1 \sim$ 2 in $70 \%$ and $\geq 3$ in $51.2 \%$. Moreover, high school 
students in Korea are under considerably high stress regarding academic performance, career issues, and pressure to achieve outstanding grades, in a competitive educational environment for university entrance examination $^{3,4)}$. The level of stress is further increased by insufficient spare time, parental pressure, and expectations of academic performance at home ${ }^{5)}$. The result of examining the level of entrance exam stress in high school students per sub-area, showed that the score for examtension/poor-grade stress was 3.08 points out of 5 points' for parental-pressure stress was 2.56 points, and for insufficient-spare-time, stress was 2.52-point ${ }^{6)}$. As such, excessive stress and poor oral health behaviors exhibited by students may exert a negative influence on their oral health status by increasing the frequency of experiencing varying symptoms of oral diseases.

In a study on adolescents, the frequency of caries on permanent teeth increased in students under stress ${ }^{7)}$, and the symptoms of oral diseases experienced by adolescents in the recent year, were reported to include pain during mastication in $85.5 \%$, pain on the tongue or the oral mucosa in $81.1 \%$, and halitosis in $86.0 \%^{8)}$. Similarly, in the case of third-year female high school students, an increase in the level of entrance exam stress led to low oral health care self-efficacy including tooth brushing, flossing, and visiting a dental clinic $^{9)}$, and an increase in the level of entrance exam stress in high school students, in general, led to a high level of subjective awareness on oral diseases such as dental caries, temporomandibular disorder, mucosal disease, and xerostomia ${ }^{6}$. The reports thus indicated a need for proper oral health education and appropriate ways of stress relief, as stress was shown to lead to various symptoms of oral diseases recognized by the students themselves. Notably, as subjective oral health awareness in students shows a close association with oral health enhancing behaviors ${ }^{10)}$ and the quality of life including oral cavity functions and food intake quantity and quality ${ }^{11)}$, positive subjective awareness on oral health are crucial in the oral health care of high school students more susceptible to oral diseases.

Numerous studies have been conducted regarding oral health behaviors, the relationship between oral health and stress, and the subjective oral health status in relation to stress in high school students. However, there is a general lack of studies on the influence of the level of entrance exam stress in high school students on oral health behaviors and subjective oral health status.

This study thus aims to investigate the effects of entrance exam stress on oral health behaviors and subjective oral health status in female high school students to provide the fundamental data to explore the proper ways of oral health care in high school students.

\section{Materials and Methods}

\section{Subjects and data collection}

The subjects were first-, second-, and third-year female high school students in Gwangju region. Convenient sampling was used, and all subjects were those who expressed the understanding of the questionnaire contents and study purposes while voluntarily participating in the study with a written consent, and capable of verbal communication. $\mathrm{G}^{*}$ power 3.1.9.4 program was used to analyze the number of subjects. For multiple regression analysis, the following condition was applied: significance level 0.05 , testing power $95 \%$, effect size 0.15 , and 13 predictors. The final number of subjects required for such analysis was 189; however, considering the dropout rate, the final number was determined as 250 . A self-reported questionnaire was administered during the period of July $15 \sim 19,2019$, and after excluding the 34 survey sheets with inadequate responses, 216 sheets were used for the final analysis.

\section{Survey tool}

The questionnaire used in this study was constructed based on a consultation with two dental hygiene professors and two high school teachers. For general characteristics of the study subjects, three questions were devised regarding the school year, academic performance, and family income. For the oral health behaviors, five questions were devised regarding the intake of toothdecaying snacks and tooth-decaying beverages, the use of an auxiliary oral hygiene product, the average number of daily brushing, and the visit to a dental clinic in the recent year. For the entrance exam stress and subjective oral health 
status, 24 and 26 questions were devised respectively.

\section{1) Entrance exam stress}

For the entrance exam stress, the tool in Kim and Han ${ }^{6}$ was applied, where the sub-areas included 9 questions on patients pressure stress, 6 questions on tension for exam/poor result stress, 6 questions on insufficient spare time stress, and 3 questions on future uncertainty stress. Likert 5-point scale was used, where an increase in the score indicated an increase in the level of entrance exam stress. The level of confidence was as follows: Cronbach's $\alpha=0.91$ for patients pressure stress, Cronbach's $\alpha=0.66$ for tension for exam/poor result stress, Cronbach's $\alpha=0.76$ for insufficient spare time stress, and Cronbach's $\alpha=0.89$ for future uncertainty stress.

\section{2) Subjective oral health status}

For the subjective oral health status, students' selfrecognized symptoms in the past six months were examined, and the questionnaire in $\mathrm{Kim}$ and $\mathrm{Han}^{6)}$ was reconstructed to fit the goals of this study: 6 questions on dental caries, 9 questions on temporomandibular disorder, 4 questions on oral mucosal disease, 4 questions on xerostomia, and 3 questions on bad breath. Likert 5-point scale was used, where an increase in the score indicated an increase in the level of self-awareness by the student on the relevant disease. The level of confidence was as follows: Cronbach's $\alpha=0.78$ for dental caries, Cronbach's $\alpha=0.88$ for temporomandibular disorder, Cronbach's $\alpha=0.70$ for oral mucosal disease, Cronbach's $\alpha=0.78$ for

Table 1. The General Characteristics of the Subjects

\begin{tabular}{lc}
\hline \multicolumn{1}{c}{ Variable } & $\mathrm{n}(\%)$ \\
\hline Grade & $72(33.3)$ \\
1st grade & $72(33.3)$ \\
2nd grade & $72(33.3)$ \\
3rd grade & \\
Academic performance & $52(24.1)$ \\
Top & $126(58.3)$ \\
Middle & $38(17.6)$ \\
Down & \\
Family income level & $19(8.8)$ \\
High & $177(81.9)$ \\
Middle & $20(9.3)$ \\
Low & \\
\hline
\end{tabular}

xerostomia, and Cronbach's $\alpha=0.86$ for bad breath.

\section{Data analysis}

Frequency analysis was performed for general characteristics and oral health behaviors, and for comparatively analyzing the differences in the average levels of entrance exam stress and subjective oral health status based on the general characteristics and oral health behaviors, independent t-test and one way ANOVA were performed. As the post-hoc test, the Scheffe test was used. The correlation between entrance exam stress and subjective oral health status was analyzed using Pearson's correlation coefficient. Multiple regression analysis was performed to determine the factors influencing the subjective oral health status. IBM SPSS for Window version 21.0 (IBM Corp., Armonk, NY, USA) was used to analyze all data, with the level of significance set to $\alpha=0.05$.

\section{Results}

\section{General characteristics and oral health behaviors}

The number of students in each year (first-, second-, and third-years) was $72(33.3 \%)$, while for the academic performance, $58.3 \%$ of the students were in the middle and $24.1 \%$ were in the upper level, and for the family income, $81.9 \%$, which is the largest proportion, were in the middle level (Table 1). The frequency of the intake of sugary,

Table 2. Oral Health Behavior of the Subjects

\begin{tabular}{lr}
\hline \multicolumn{1}{c}{ Variable } & $\mathrm{n}(\%)$ \\
\hline Number of caries snacks per week & \\
$\quad \leq 3$ & $76(35.2)$ \\
$\geq 4$ & $140(64.8)$ \\
Number of caries drinks per week & \\
$\quad \leq 3$ & $133(61.6)$ \\
$\quad \geq 4$ & $83(38.4)$ \\
Use of oral hygiene products & $68(31.5)$ \\
$\quad$ Yes & $148(68.5)$ \\
$\quad$ No & \\
Number of daily brushing & $46(21.3)$ \\
$\quad \leq 2$ & $170(78.7)$ \\
$\quad \geq 3$ & \\
Dental visit experience within the last year & $152(70.4)$ \\
$\quad$ Yes & $64(29.6)$ \\
No &
\end{tabular}




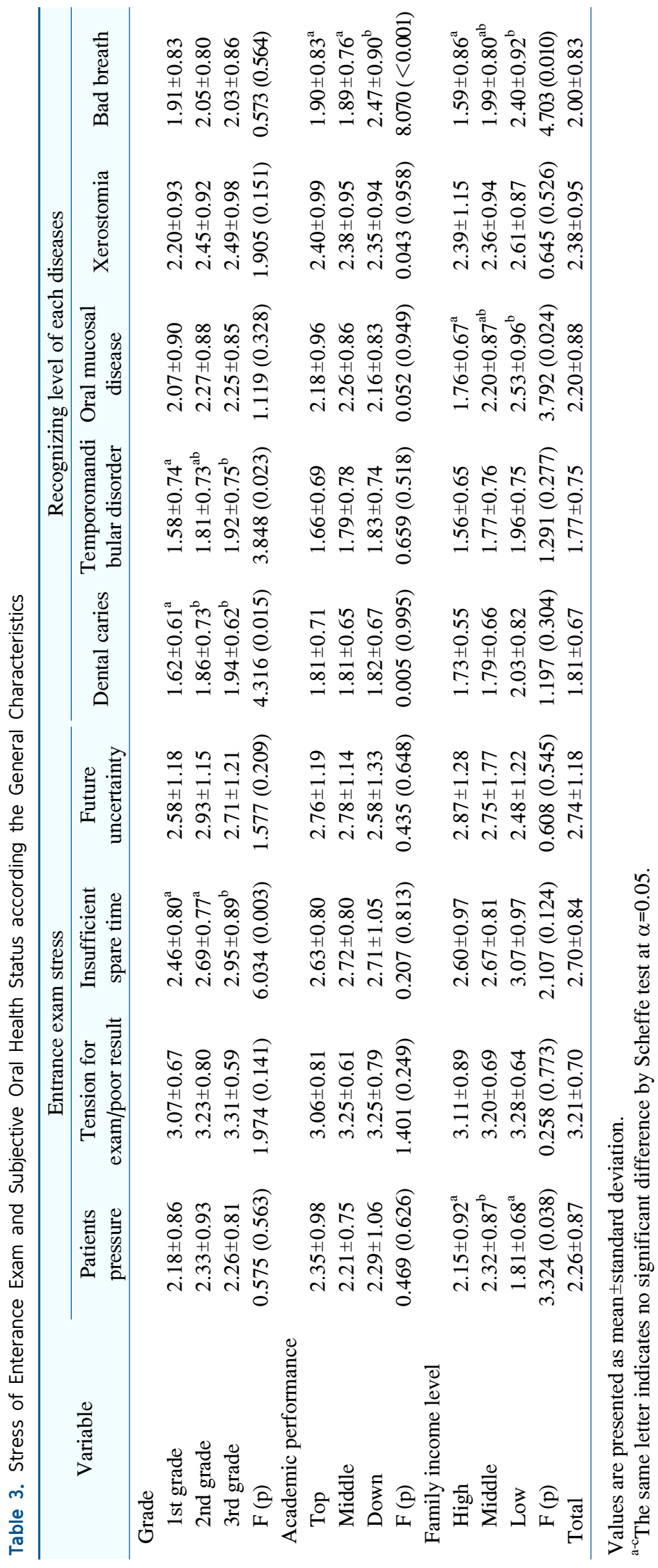


tooth-decaying foods such as crisps, candy, and cake in a week was $\geq 4$ times in $64.8 \%$ of the students, while the frequency of the intake of carbonated or fizzy drinks was $\leq 3$ times in $61.6 \%$, the largest proportion of students. Moreover, $68.5 \%$ lacked the experience of using an auxiliary oral hygiene product, and the average number of daily brushing was $\geq 3$ times in $78.7 \%$, and $70.4 \%$ of the students had the experience of visiting a dental clinic in the past year (Table 2).

\section{Analysis of the entrance exam stress and subjective oral health status based on general characteristics}

The result of analyzing the entrance exam stress and subjective oral health status based on general characteristics is presented in Table 3. Among the sub-areas of entrance exam stress, the highest score was 3.21 shown in tension for exam/poor result, followed by 2.74 in future uncertainty, 2.70 in insufficient spare time, and 2.26 in patients pressure. The level of patients pressure stress was significantly high for the students in the middle family income level $(\mathrm{p}<0.05)$. The level of insufficient spare time stress was significantly higher in third-year students than those in the first or second year $(p<0.01)$. For students' self-recognized symptoms regarding subjective oral health status, the highest score was 2.38 shown for xerostomia, followed by 2.20 for oral mucosal disease, 2.00 for bad breath, 1.81 for dental caries, and 1.77 for temporomandibular disorder. The scores for dental caries and temporomandibular disorder were significantly higher in third-year students than those in the first or second year $(p<0.05)$, and the score for oral mucosal disease indicated higher self-awareness for the students in the low family income level $(\mathrm{p}<0.05)$, while the score for bad breath indicated higher self-awareness for the students with down academic performance $(\mathrm{p}<0.001)$ and for those in the low family income level $(\mathrm{p}<0.05)$.

\section{Analysis of the oral health behaviors and subjective oral health status}

The result of analyzing the oral health behaviors and subjective oral health status is presented in Table 4. The

Table 4. Subjective Oral Health Status Analysis according to Oral Health Behavior

\begin{tabular}{|c|c|c|c|c|c|}
\hline \multirow[b]{2}{*}{ Variable } & \multicolumn{5}{|c|}{ Recognizing level of each diseases } \\
\hline & Dental caries & $\begin{array}{c}\text { Temporomandibular } \\
\text { disorder }\end{array}$ & $\begin{array}{c}\text { Oral mucosal } \\
\text { disease }\end{array}$ & Xerostomia & Bad breath \\
\hline \multicolumn{6}{|c|}{ Number of caries snacks per week } \\
\hline$\leq 3$ & $1.68 \pm 0.61$ & $1.66 \pm 0.73$ & $2.16 \pm 0.88$ & $2.28 \pm 0.94$ & $1.91 \pm 0.90$ \\
\hline$\geq 4$ & $1.88 \pm 0.69$ & $1.83 \pm 0.76$ & $2.21 \pm 0.88$ & $2.44 \pm 0.96$ & $2.04 \pm 0.78$ \\
\hline $\mathrm{t}(\mathrm{p})$ & $-2.069(0.040)$ & $-1.578(0.116)$ & $-0.464(0.643)$ & $-1.135(0.258)$ & $-1.102(0.272)$ \\
\hline \multicolumn{6}{|c|}{ Number of caries drinks per week } \\
\hline$\leq 3$ & $1.80 \pm 0.72$ & $1.71 \pm 0.73$ & $2.17 \pm 0.88$ & $2.23 \pm 0.88$ & $2.01 \pm 0.87$ \\
\hline$\geq 4$ & $1.82 \pm 0.58$ & $1.86 \pm 0.78$ & $2.24 \pm 0.87$ & $2.62 \pm 1.01$ & $1.97 \pm 0.76$ \\
\hline $\mathrm{t}(\mathrm{p})$ & $-0.135(0.892)$ & $-1.326(0.186)$ & $-0.511(0.610)$ & $-2.892(0.004)$ & $0.348(0.728)$ \\
\hline \multicolumn{6}{|c|}{ Use of oral hygiene products } \\
\hline Yes & $1.76 \pm 0.61$ & $1.90 \pm 0.84$ & $2.30 \pm 0.89$ & $2.43 \pm 1.00$ & $2.07 \pm 0.82$ \\
\hline No & $1.83 \pm 0.69$ & $1.71 \pm 0.70$ & $2.15 \pm 0.87$ & $2.36 \pm 0.93$ & $1.96 \pm 0.83$ \\
\hline $\mathrm{t}(\mathrm{p})$ & $-0.626(0.532)$ & $1.784(0.076)$ & $1.241(0.216)$ & $0.527(0.599)$ & $0.917(0.360)$ \\
\hline \multicolumn{6}{|l|}{ Number of daily brushing } \\
\hline$\leq 2$ & $1.78 \pm 0.58$ & $1.81 \pm 0.69$ & $2.26 \pm 0.88$ & $2.46 \pm 1.06$ & $2.33 \pm 0.79$ \\
\hline$\geq 3$ & $1.81 \pm 0.69$ & $1.75 \pm 0.77$ & $2.18 \pm 0.88$ & $2.36 \pm 0.92$ & $1.90 \pm 0.82$ \\
\hline $\mathrm{t}(\mathrm{p})$ & $-0.304(0.761)$ & $0.439(0.661)$ & $0.535(0.593)$ & $0.629(0.530)$ & $3.083(0.002)$ \\
\hline \multicolumn{6}{|c|}{ Dental visit experience within the last year } \\
\hline Yes & $1.77 \pm 0.67$ & $1.78 \pm 0.78$ & $2.15 \pm 0.90$ & $2.34 \pm 0.93$ & $1.97 \pm 0.82$ \\
\hline No & $1.88 \pm 0.66$ & $1.73 \pm 0.68$ & $2.31 \pm 0.83$ & $2.47 \pm 1.01$ & $2.04 \pm 0.85$ \\
\hline $\mathrm{t}(\mathrm{p})$ & $-1.005(0.316)$ & $0.500(0.800)$ & $-1.228(0.221)$ & $-0.892(0.373)$ & $-0.512(0.609)$ \\
\hline
\end{tabular}

Values are presented as mean \pm standard deviation. 
self-awareness of dental caries was higher for the students whose intake of tooth-decaying snacks (per week) was 4 or more times $(p<0.05)$, and the self-awareness of xerostomia was higher for the students whose intake of tooth-decaying beverages (per week) was 4 or more times $(\mathrm{p}<0.01)$. The self-awareness of bad breath was shown to be higher for the students whose average number of daily brushing was $\leq 2$ times $(\mathrm{p}<0.01)$.

\section{Correlation between entrance exam stress and subjective oral health status}

The result of analyzing the correlation between entrance exam stress and subjective oral health status in subjects is presented in Table 5. A positive correlation was found between dental caries and the following stress areas: patients pressure $(\mathrm{r}=0.243, \mathrm{p}<0.001)$, tension for exam/ poor result $(\mathrm{r}=0.224, \mathrm{p}<0.01)$, and insufficient spare time $(\mathrm{r}=0.252, \mathrm{p}<0.001)$. For temporomandibular disorder, $\mathrm{a}$ positive correlation was found with patients pressure $(\mathrm{r}=0.146, \mathrm{p}<0.05)$, tension for exam/poor result $(\mathrm{r}=0.145$, $\mathrm{p}<0.05)$, and insufficient spare time $(\mathrm{r}=0.283, \mathrm{p}<0.001)$, while for oral mucosal disease, a positive correlation was found with patients pressure $(r=0.143, p<0.05)$, tension for exam/poor result $(r=0.206, p<0.01)$, and insufficient spare time $(\mathrm{r}=0.260, \mathrm{p}<0.001)$. A positive correlation was found between xerostomia and patients pressure $(r=0.226$, $\mathrm{p}<0.01)$, tension for exam/poor result $(\mathrm{r}=0.234, \mathrm{p}<0.01)$, insufficient spare time $(\mathrm{r}=0.381, \mathrm{p}<0.001)$, while for bad breath, a positive correlation was found with tension for exam/poor result $(\mathrm{r}=0.209, \mathrm{p}<0.01)$ and insufficient spare time $(\mathrm{r}=0.242, \mathrm{p}<0.001)$.

\section{Effects on subjective oral health status}

To examine the factors influencing the subjective oral health status, multiple regression analysis was carried out and the result is presented in Table 6. The method of variable selection was used for the input so as to analyze the level of influence of each independent variable

Table 5. Correlation between Entrance Exam Stress and Subjective Oral Health Status

\begin{tabular}{lccccc}
\hline \multicolumn{1}{c}{ Variable } & Dental caries & $\begin{array}{c}\text { Temporomandibular } \\
\text { disorder }\end{array}$ & $\begin{array}{c}\text { Oral mucosal } \\
\text { disease }\end{array}$ & Xerostomia & Bad breath \\
\hline Patients pressure & $0.243^{* * *}$ & $0.146^{*}$ & $0.143^{*}$ & $0.226^{* *}$ & 0.108 \\
Tension for exam/poor result & $0.224^{* *}$ & $0.145^{*}$ & $0.206^{* *}$ & $0.234^{* *}$ & $0.209^{* *}$ \\
Insufficient spare time & $0.252^{* * *}$ & $0.283^{* * *}$ & $0.260^{* * *}$ & $0.381^{* * *}$ & $0.242^{* * *}$ \\
Future uncertainly & 0.115 & 0.108 & 0.003 & 0.099 & 0.045 \\
\hline
\end{tabular}

${ }^{*} \mathrm{p}<0.05,{ }^{* *} \mathrm{p}<0.01,{ }^{* * *} \mathrm{p}<0.001$ by pearson's correlation analysis.

Table 6. Effects on Subjective Oral Health Status

\begin{tabular}{|c|c|c|c|c|c|c|c|}
\hline Variable & B & $\begin{array}{c}\text { Standard } \\
\text { error }\end{array}$ & $\beta$ & $\mathrm{t}$ & p-value & $\mathrm{F}(\mathrm{p})$ & $\begin{array}{c}\text { Adjusted } \\
\mathrm{R}^{2}\end{array}$ \\
\hline Dental caries & & & & & & $3.694(<0.001)$ & 0.149 \\
\hline Grade (3rd year) & 0.228 & 0.111 & 0.160 & 2.054 & 0.041 & & \\
\hline Patients pressure & 0.156 & 0.059 & 0.202 & 2.629 & 0.009 & & \\
\hline Temporomandibular disorder & & & & & & $2.052(0.019)$ & 0.060 \\
\hline Insufficient spare time & 0.202 & 0.079 & 0.227 & 2.558 & 0.011 & & \\
\hline Oral mucosal disease & & & & & & $2.229(0.010)$ & 0.069 \\
\hline Family income level (high) & -0.451 & 0.214 & -0.145 & -2.106 & 0.036 & & \\
\hline Insufficient spare time & 0.198 & 0.092 & 0.190 & 2.162 & 0.032 & & \\
\hline Xerostomia & & & & & & $3.191(<0.001)$ & 0.117 \\
\hline Insufficient spare time & 0.387 & 0.097 & 0.342 & 3.989 & $<0.001$ & & \\
\hline Bad breath & & & & & & $3.704(<0.001)$ & 0.141 \\
\hline Academic performance (down) & 0.504 & 0.150 & 0.231 & 3.367 & 0.001 & & \\
\hline Number of daily brushing $(\leq 2)$ & 0.354 & 0.130 & 0.175 & 2.718 & 0.007 & & \\
\hline Insufficient spare time & 0.181 & 0.083 & 0.184 & 2.173 & 0.031 & & \\
\hline
\end{tabular}


reflected in the regression model. All optimized models showed statistical significance $(\mathrm{p}<0.05)$. A significantly higher level of awareness was shown for dental caries in third-year students $(\beta=0.160)$; for oral mucosal disease, in the students of lower family income level $(\beta=-0.145)$; for bad breath, in the students with lower academic performance ( $\beta=0.231)$ and $\leq 2$ times daily brushing $(\beta=0.175)$.

\section{Discussion}

Social circumstances in Korea regarding university admission implies that high school students are under a considerable level of entrance exam stress, and during the period, an increase in the level of stress leads to poor oral hygiene management in adolescents as well as a high rate of contracting oral diseases such as dental caries and periodontal diseases. The level of stress could thus be a critical factor affecting the oral health behaviors in adolescents ${ }^{12}$. Furthermore, an increase in entrance exam stress leads to increased subjective awareness on oral diseases in high school students ${ }^{6}$, and depending on the practice of oral health behaviors, the level of stress may exert a direct influence on the subjective oral health status ${ }^{13)}$. Therefore, this study investigated the effects of the entrance exam stress on oral health behaviors and subjective oral health status in female high school students to provide fundamental data for exploring proper ways of oral health care in high school students.

In this study, the level of entrance exam stress was the highest for the sub-area of tension for exam/poor result (3.21-point), followed by future uncertainty 2.74-point, insufficient spare time 2.70-point, and patients pressure 2.26-point. Kim and $\mathrm{Han}^{6}$, a study targeting high school students, and Cho and Chung', a study targeting female high school students, also showed that the level of examtension/poor-grade stress was the highest, despite slight differences in each sub-area of entrance exam stress according to year and sex.

The subjective awareness of dental caries was significantly higher in students whose weekly intake of tooth-decaying snacks was $\geq 4$ times. The result of multiple regression analysis showed that the subjective awareness of dental caries increased in third-year students and with an increase in patients pressure stress among the sub-areas of entrance exam stress. A high level of stress in high school students led to the increased frequency of caries on permanent tooth $^{7)}$, and in a study investigating the effects of stress on dietary habits of high school students, a high level of stress led to significantly low probability of the student taking protective foods but significantly high probability of the student taking tooth-decaying foods ${ }^{14)}$. Ji and Lee ${ }^{15)}$ also showed a positive correlation between stress and the behavior of eating sugary foods in high school students. As observed, stress led to an increase in the intake of tooth-decaying foods and in the probability of inducing dental caries in high school students, which may account for increased subjective awareness of dental caries in this study. Thus, as a way of stress relief, appropriate oral health education is necessary so that students may form a proper dietary habit to prevent frequent intake of tooth-decaying snacks and proactively manage their oral hygiene after the intake of snacks.

In a study on third-year high school students, $71.3 \%$ responded that they had experienced at least one or more symptoms among the 10 listed symptoms related to temporomandibular disorder, while $41.7 \%$ responded two or more symptoms and $25.7 \%$ responded three or more symptoms. For the difference in the number of students with temporomandibular disorder symptoms according to the level of stress, statistically significant figures were obtained from students who had depressive feelings in daily life, those who had anxiety or tension, and those who reported a high level of stress since several years ago ${ }^{16}$.

Although a direct comparison is difficult due to the use of different stress measurement tools, the symptoms of temporomandibular disorder in this study were also experienced to a greater extent by third-year students than those in the first- or second-year, with statistical significance. The result of multiple regression analysis showed that high subjective awareness of the symptoms of temporomandibular disorder was under significant influence of a high level of insufficient spare time stress among the sub-areas of entrance exam stress. This coincided with the result of Kim and $\mathrm{Han}^{6}$.

Furthermore, the level of insufficient spare time stress was found to be significantly higher in third-year students 
than first- or second-year students. The high school students in the third-year spend most of their time at school in preparation for the university entrance exam, and in reality, are not given sufficient spare time for hobbies or activities to release stress, which seems to account for the high level of insufficient-spare-time stress. However, when such stress increases further to reach a serious level, prolonged muscle contraction results in interfering with normal blood flow in the muscle tissue, causing pain, and affecting the position of temporomandibular joint, leading to aggravated symptoms of temporomandibular disorder ${ }^{17,18)}$. Thus, students should be given a chance to use an appropriate amount of spare time to release stress. The factors that influence the number of temporomandibular disorder symptoms were reported to include biting lips or cheeks, chewing gums, chin propping, and hard food eating habit ${ }^{15)}$, and based on this, students showing a severe level of insufficient spare time stress should be given an education to refrain from such habits to prevent temporomandibular disorder.

Subjective awareness of the symptoms of oral mucosal disease was found to be under a greater influence of the family income level when it is low and of the insufficient spare time stress when it is high. However, Kim and $\mathrm{Han}^{6}$, a study targeting high school students, reported a contrasting result where the exam-tension/poor-grade stress was the main stress factor related to oral mucosal health, while family income level showed no significant difference.

In a study on the association between subjective awareness of stress and the experience of oral symptoms in adolescents in Korea, the symptoms of soft tissue diseases showed a significantly higher level by 2.55 times if the stress was of low level and by 1.59 times if the stress was of middle level ${ }^{19)}$. The level of stress was also found to have a significant direct influence on the frequency of oral mucosal disease ${ }^{20)}$. Accordingly, a specific method to reduce the insufficient spare time stress in high school students should be sought, as frequent occurrence of stomatitis and cheilitis on the tongue, lips, and oral mucosa would cause difficulty ingesting foods and managing oral hygiene.

The subjective awareness of halitosis was found to be under a significantly greater influence of the average number of daily brushing when it is $\leq 2$ times or lower and of the insufficient-spare-time stress when it is high. In a previous study investigating the factors related to halitosis awareness in high school students, $37.5 \%$ of students who brush their teeth once a day responded they had been aware of halitosis, and $53.8 \%$ of students who brush their teeth twice a day responded that they had sometimes been aware of halitosis. The halitosis awareness and the frequency of brushing showed a negative correlation, where the low frequency of brushing led to a high level of halitosis awareness ${ }^{21)}$. This agreed with the result in this study, and proper brushing was indicated to be crucial in reducing the self-recognized halitosis symptoms in high school students. Thus, students should be given proper brushing training including the number of daily brushing, the brushing method, and the adequate brushing time.

In a study targeting a group of adults, stress was the most important factor influencing halitosis. An increase in the level of stress by 1 resulted in 232.354-fold increase of dimethyl sulfide $\left.\left(\left(\mathrm{CH}_{3}\right)_{2}\right) \mathrm{S}\right)$ among the volatile sulfur compounds used to assess the level of halitosis ${ }^{22)}$. Although a simple comparison is difficult due to the differences in study subjects, stress measurement tool, and halitosis evaluation tool, the previous study suggested a need for developing a practical method to reduce the insufficient-spare-time stress that had been determined as a factor influencing the halitosis awareness in high school students in this study. However, a different result was reported in $\mathrm{Kim}$ and $\mathrm{Han}^{6}$, where no significant differences were found with respect to the entrance exam stress and the subjective awareness of halitosis in high school students. In future, a follow-up study should focus on the volatile sulfur compounds measured for the students with subjective awareness of halitosis and its relation to the entrance exam stress.

The limitations of this study are as follows: i) As required by the characteristics of this study, a self-reported questionnaire was applied, implicating a possibility of subjective bias according to the individual student learning environment and exam period; ii) A group of female high school students in a single region was investigated, so that 
it is difficult to generalize the results in this study; iii) Due to the lack of studies related to this study, comparisons were made with the studies regarding the stress in high school students, adolescents, or adults.

Nevertheless, the study has identified the most influential factor on oral health status among the sub-areas of the entrance exam stress regarding the oral health behaviors in female high school students, based on which it is anticipated that the findings will prove useful as the fundamental data for exploring the proper ways of oral health care in high school students. Follow-up studies should continue to investigate the incidence of oral diseases in students while focusing on those with a high level of entrance exam stress, based on the analysis of objective oral health examination data.

\section{Notes}

\section{Conflict of interest}

No potential conflict of interest relevant to this article was reported.

\section{Ethical approval}

This study was approved by the institutional review board of Honam University (IRB No. 1041223-201906HR-07).

\section{Author contributions}

Conceptualization, Data acquisition, Formal analysis, Funding, Supervision, Writing-original draft, Writingreview \& editing: Kyung-Yi Chung.

\section{ORCID}

Kyung-Yi Chung, https://orcid.org/0000-0001-6864-1670

\section{Acknowledgements}

This study was supported by research fund from Honam University, 2019.

\section{References}

1. Park HJ, Lee JH: The relation between periodontal condition and dental health behaviors among Korean adolescents.
Proceedings of the KAIS Fall Conference. J Korea Acad-Ind Coop Soc 946-948, 2009.

2. Nam JY, Kim HJ: The influence of oral symptom recognition and oral care behavior on cases of dental caries in high school student. Asia-Pac J Multimed Serv Converg Art Humanit Sociol 6: 141-152, 2016.

3. Lee MN, Lee HY: Analysis of dietary habits of boarding high school students in Gangwon according to gender and stress levels. J Korean Soc Food Cult 28: 48-56, 2013. https://doi.org/10.7318/KJFC/2013.28.1.048

4. Seo JY, Kim MY: Stress, physical symptoms, and coping styles of high school students. J Korean Acad Child Health Nurs 12: 470-477, 2006.

5. Lee HJ, Park YS: The influence of high school students' entrance exam stress on their mental health. J Korean Soc Sch Community Health Educ 8: 43-54, 2007.

6. Kim SR, Han SJ: The relationship between perceived oral health status and entrance exam stress levels in high school students. J Dent Hyg Sci 15: 509-517, 2015. https://doi.org/10.17135/jdhs.2015.15.4.509

7. Ma JK, Cho MJ: Association of sleep time, stress, and depression with the oral health status of Korean adolescents. J Korean Acad Oral Health 40: 178-182, 2016. https://doi.org/10.11149/jkaoh.2016.40.3.178

8. Chun JY, Lee KH: The correlation between oral health factors and mental health factors in Korean adolescents. J Korean Soc Dent Hyg 17: 669-680, 2017. https://doi.org/10.13065/jksdh.2017.17.04.669

9. Cho HE, Chung KY: The relationship between enterance exam stress and oral care self-efficacy in 3rd year girl high school students. J Korea Entertain Ind Assoc 13: 551-561, 2019. https://doi.org/10.21184/jkeia.2019.12.13.8.551

10. Lim SA: Convergence factors of subjective oral health awareness perception on oral health improvement behavior in some university students. J Korea Converg Soc 8: 167-175, 2017.

https://doi.org/10.15207/JKCS.2017.8.11.167

11. Chang IJ, Jeong SH, Park YA, Lee HK, Song KB: Association between denture satisfaction and perceived oral health among the elderly with removable denture. J Korean Acad Dent Health 30: 360-369, 2006.

12. Yu MS, Goo KM, Kim JS: A study on the oral health behavior of high school students in Jeolla-bukdo. J Dent Hyg 
Sci 9: 225-230, 2009.

13. Park HJ, Kim HW, Ko SY, Lee JH: Moderating effects of oral health behaviors on the relation between daily stress and oral health status in Korean adolescents. Korean Public Health Res 41: 81-93, 2015.

14. Park JY, Kim SM: Effects of stress perception level on dietary habits and oral health behaviors in adolescents. J Dent Hyg Sci 16: 111-117, 2016. https://doi.org/10.17135/jdhs.2016.16.2.111

15. Ji MG, Lee MR: The convergence relationship on stress, sugar intake behaviors, and oral health status in high school students. J Converg Inf Technol 9: 118-129, 2019. https://doi.org/10.22156/CS4SMB.2019.9.4.118

16. Cho MS, Yi SJ: Self-report symptoms for temporomandibular disorder and related factors in the high school third grade students. J Korean Soc Dent Hyg 11: 853-862, 2011.

17. Lee JH, Choi JM: A study on the temporomandibular joint disorder and school life stress of high school student by department. J Dent Hyg Sci 7: 179-185, 2007.
18. Park HS: An epidemiologic study of symptoms of temporomandibular disorders in Korean college students. J Oral Med Pain 32: 91-104, 2007.

19. Han YJ, Kim HS, Ryu SY: Association with oral symptom experiences by level of subjective stress recognition in the Korean adolescents. J Korean Soc Dent Hyg 17: 465-478, 2017.

https://doi.org/10.13065/jksdh.2017.17.03.465

20. Hong MH: The influence of stress on oral mucosal disease, dry mouth and stress symptoms in adults. J Korean Soc Dent Hyg 13: 589-596, 2013. https://doi.org/10.13065/iksdh.2013.13.4.589

21. Jung YS, Lee MR: Self-perception of halitosis and the halitosis-related factors for high school student. J Korea Entertain Ind Assoc 9: 227-234, 2015. https://doi.org/10.21184/jkeia.2015.06.9.2.227

22. Kim KE, Han JH: Correlation among halitosis, xerostomia and stress in adults. J Dent Hyg Sci 16: 370-377, 2016. https://doi.org/10.17135/jdhs.2016.16.5.370 